\title{
Intramuscular Cysts at the Proximal Calf: Two Case Reports and a Systematic Review of Literature
}

\author{
Ning Fan ${ }^{\mathrm{a}}$, Yongchen Zheng ${ }^{\mathrm{a}}$, Chenggang Yang, ${ }^{\mathrm{a}}$, Jiusheng Hea, Yanmei Liu ${ }^{\mathrm{a}}$, \\ Qing Zhao ${ }^{\mathrm{a}}$, Jinwei Wang ${ }^{\mathrm{a}}$
}

\begin{abstract}
Intramuscular cysts at the proximal calf are rare; however, their etiology is diverse, making accurate diagnosis and treatment difficult. To our knowledge, there are no previous publications in literature about intramuscular cysts at the proximal calf, arising from different origins. This article describes two rare cases with intramuscular cysts. Case 1 was diagnosed as an intramuscular ganglion cyst arising from the proximal gastrocnemius medialis muscle, and arthroscopic examination as well as open cyst excision was performed. Case 2 was another type, which has never been reported in the literature. It was diagnosed as diffuse pigmented villonodular synovitis (PVNS), with the intramuscular cyst arising from PVNS, and only arthroscopic synovectomy was performed. The diagnoses for these cases were confirmed by histopathology. In addition, we have summarized intramuscular cysts at the proximal calf by a systematic literature review and divided them into five types based on their origin. The five types are, intramuscular cysts arising from the muscular tissue, knee joint, proximal tibiofibular joint, the neurilemma and PVNS. The treatment of intramuscular cysts varies based on their origin; hence, it is important to identify the origin.
\end{abstract}

Keywords: Cyst; Gastrocnemius muscle; Etiology; Therapy

\section{Introduction}

Many types of cysts can occur around the knee and the most frequently encountered cystic masses include Baker's cysts, cruciate ligament ganglia and meniscal cysts $[1,2]$. However, intramuscular cysts at the proximal calf are rare, and the etiologies of these cysts are diverse. Some case reports have shown that atypical Baker's cysts [3, 4], cysts arising from the proximal tibiofibular joint (PTFJ) [5-8] and nerve sheath ganglions [9-12], can lead to intramuscular cysts at the proximal

Manuscript submitted December 10, 2017, accepted December 20, 2017

${ }^{a}$ Department of Orthopedics, Beijing Shunyi District Hospital, Beijing, China ${ }^{b}$ Corresponding Author: Chenggang Yang, Department of Orthopedics, Beijing Shunyi District Hospital, No. 3, GuangMingNanJie, Shunyi District, Beijing 101300, China. Email: fanning2015@126.com

doi: https://doi.org/10.14740/jmc2984w calf. The clinical presentation of these cysts is usually a painless mass along the calf. When the cyst exerts pressure on the nerves or blood vessels, pain, numbness, and muscle weakness might occur, or there might be tenderness and discomfort during movement $[5,6,8,13,14]$. Magnetic resonance imaging (MRI) can clearly reveal the size and position of the cysts, and identify their anatomical relationship to the joint and surrounding structures. Prior to surgery, it is important to identify whether the cyst is connected to the joint, or is associated with intraarticular disease, and to perform thorough excision, to reduce the likelihood of recurrence. Although the presentation of cystic masses arising from different origins might be similar, their treatments are different, thus underscoring the importance of accurate diagnosis.

To our knowledge, there are no previous publications about intramuscular cysts at the proximal calf, arising from different origins. To arrive at an accurate diagnosis, and decide an appropriate treatment of these cysts, we describe here two cases of rare intramuscular cysts. The first case is a ganglion cyst arising from the proximal gastrocnemius medialis muscle and the second case is about a synovial cyst arising from pigmented villonodular synovitis (PVNS), which has never been reported in the literature. In addition, we have recapitulated intramuscular cysts at the proximal calf with different etiologies based on their origin, by a systematic review of literature, to aid in accurate diagnosis and appropriate treatment. The respective patients provided their consent for the publication of these cases.

\section{Case Reports}

\section{Case 1}

A 61-year-old woman presented with a painless mass at the posterior aspect of her right knee, which had grown progressively over the last 4 months. There were no symptoms of pain or numbness in her right leg. There was no history of recent local injury or surgical treatment. On examination, there was a non-tender mass of the size of a quail egg, over the gastrocnemius medialis muscle. Slight pain was elicited on deep palpation. The overlying skin was normal. The ranges of movements of the right knee were flexion $100^{\circ}$ and extension $5^{\circ}$. Remainder of the examination was normal.

Anteroposterior radiographs revealed degenerative 

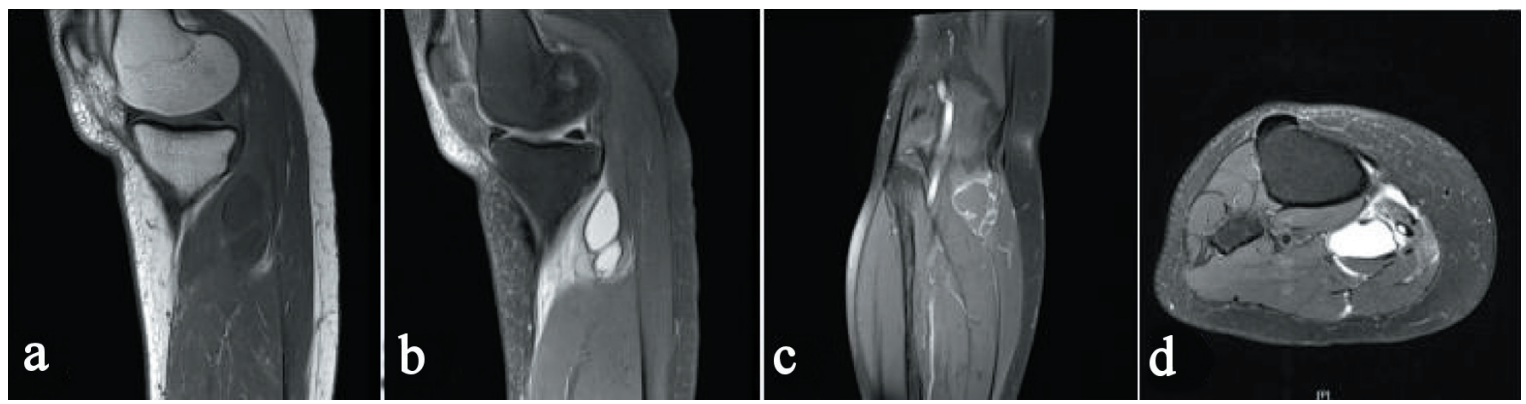

Figure 1. Preoperative magnetic resonance image of the right knee. Sagittal T1-weighted image (a), sagittal T2-weighted image (b), coronal T2-weighted image (c), and axial T2-weighted image (d) reveal a mass in the gastrocnemius medialis muscle, which does not communicate with the articular joint.

changes in the knee joint. An ultrasound (US) examination was performed for evaluation of the mass and its relation to the adjacent structures. US demonstrated a hypoechoic cystic lesion with well-defined borders and thick walls, containing scattered hyperechoic spots, located at the gastrocnemius medialis muscle. The maximum diameter was $4 \mathrm{~cm}$. MRI was performed to confirm the US findings. MRI revealed a cystic mass in the gastrocnemius medialis muscle and the mass was not attached to the knee joint (Fig. 1). The surrounding soft tissue was normal. The primary diagnosis in this case was a popliteal cyst.

A two-step surgical procedure was performed under spinal anesthesia, with the patient in supine position. First, an arthroscopic examination was performed. The anterior-lateral and anterior-medial arthroscopic portals were used to perform the procedure and no evidence of intra-articular pathology or capsular opening was found. Subsequently, a surgical exposure of the popliteal fossa was performed. Intraoperatively, the cystic mass of about $4 \times 3 \times 3 \mathrm{~cm}$ was located in the gastrocnemius medialis muscle (Fig. 2). The cyst was filled with mucoidlike material. The histological diagnosis was compatible with a ganglion cyst and a diagnosis of intramuscular ganglion cyst was made, based on these histologic findings. Postoperatively, the patient recovered completely in a few weeks. There was no recurrence or complications during 8 months of follow-up.

\section{Case 2}

A 30-year-old woman presented with gradually worsening pain and swelling around the right knee joint, since 10 months ago. There was no previous history of significant injury to the right knee and no congenital abnormalities. On examination, there was tenderness around the knee joint with a visible swelling. However, the mass was not felt on palpation. The overlying skin was normal. The ranges of the right knee movements were flexion $90^{\circ}$ and extension $0^{\circ}$. Rest of the examination was normal.

Anteroposterior radiographs revealed slight bony erosions of the lateral condyle of femur. MRI showed an extraarticular cyst measuring approximately $4 \times 1 \times 1 \mathrm{~cm}$, at the site of gastrocnemius lateralis muscle. The lesion had a wellcircumscribed margin, with low signal intensity on both T1weighted and T2-weighted images (Fig. 3). In addition, there was significant fluid in the suprapatellar bursa, and thickened synovium with low signal intensity on both T1-weighted and T2-weighted images. One of the sagittal T1-weighted images showed that the cyst wall was attached to the knee joint cap-
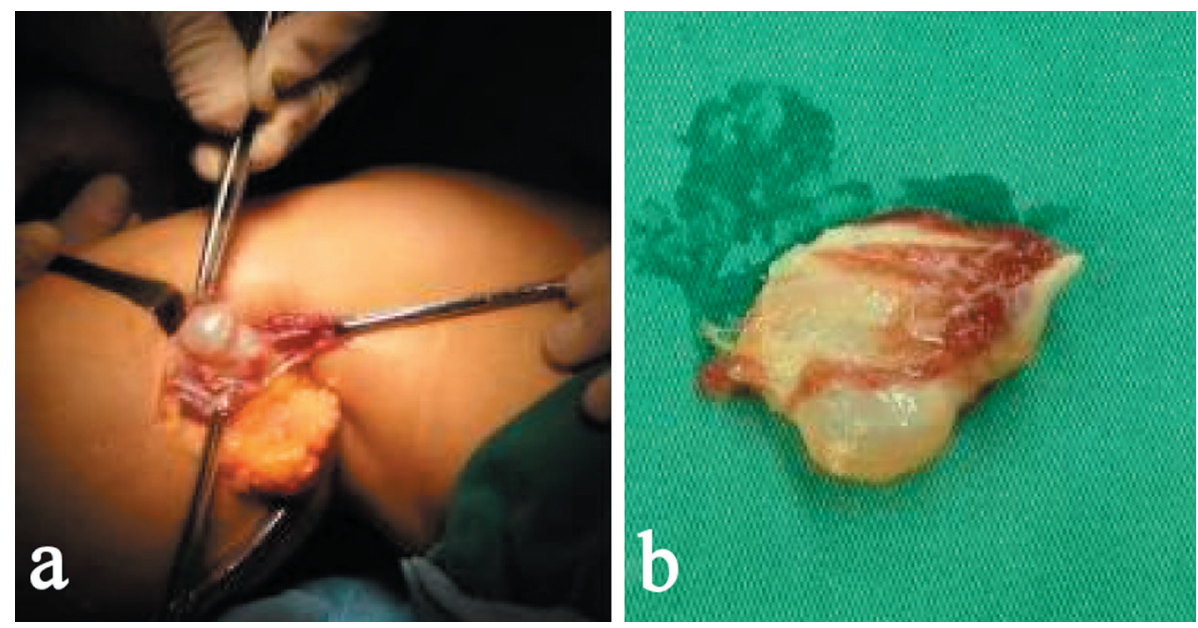

Figure 2. Intra-operative cyst located in the gastrocnemius medialis muscle (a) and partial tissue of the excised cyst (b), as the cyst ruptured partly when excised. 

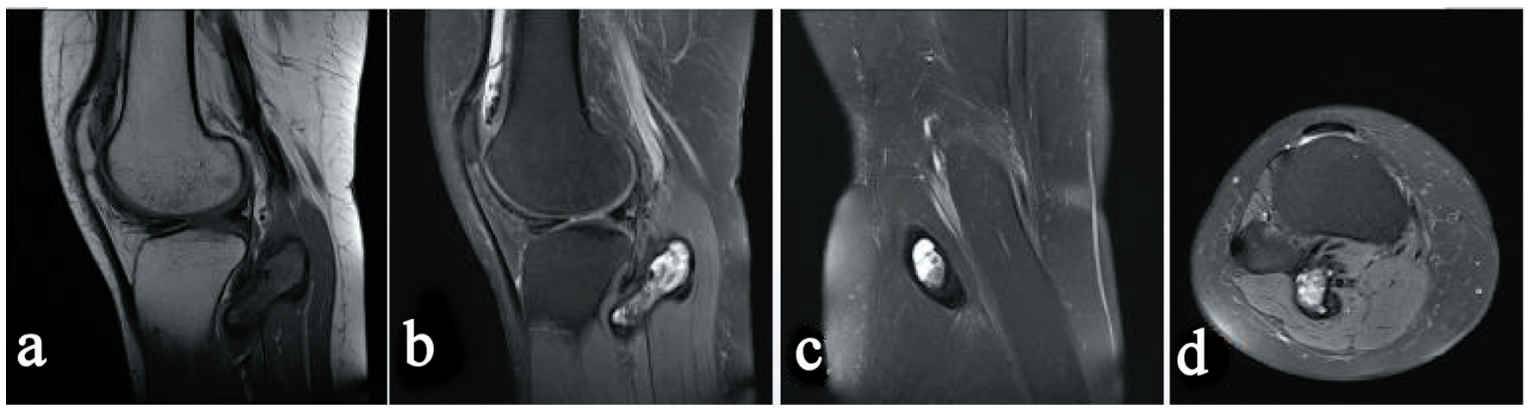

Figure 3. Preoperative magnetic resonance image (MRI) of the right knee. Sagittal T1-weighted image (a), sagittal T2-weighted image (b), coronal T2-weighted image (c), and axial T2-weighted image (d) reveal a mass in the gastrocnemius lateralis muscle. Thickened synovium with low signal intensity on both T1-weighted and T2-weighted images is found in the knee joint.

sule (Fig. 4). The surrounding soft tissue was normal. A preoperative diagnosis of diffuse PVNS was considered, and the intramuscular cyst was believed to be arising from PVNS.

With arthroscopy, using the anteromedial, anterolateral, posteromedial, and posterolateral portals, synovectomy and pathological biopsy were performed. Since there were no symptoms, no surgery or needle puncture of the intramuscular cyst was performed. Histopathology confirmed the diagnosis of diffuse PVNS. The patient was fully asymptomatic with no evidence of recurrence at the most recent 6-month follow-up after surgery.

\section{Discussion}

We have described two rare intramuscular cysts, thus highlighting the need for consideration of intramuscular ganglion cyst or intramuscular cyst arising from PVNS, in the differen-

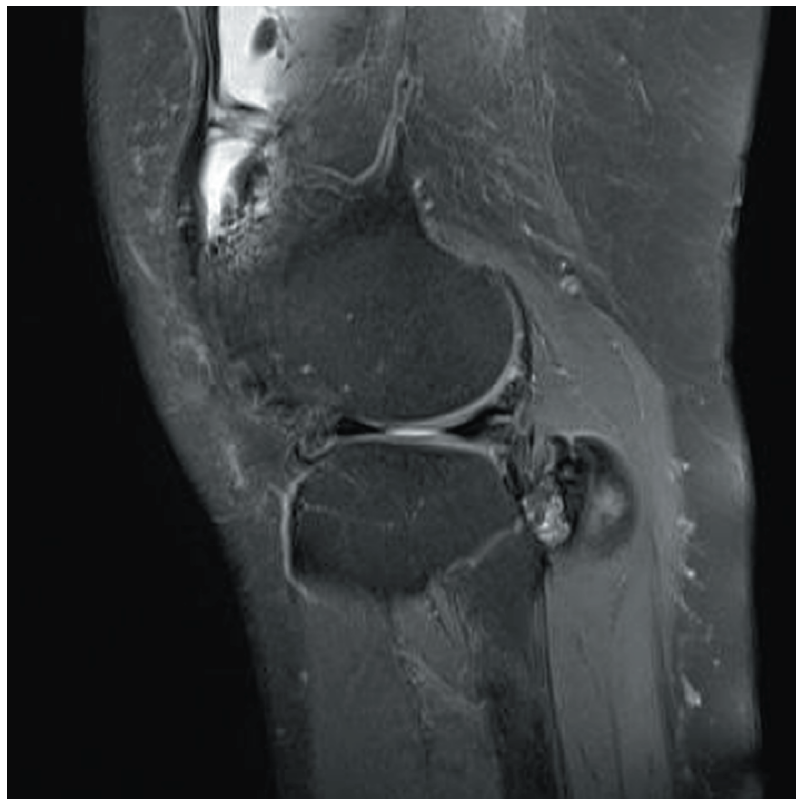

Figure 4. Preoperative magnetic resonance image (MRI) of the right knee. Sagittal T2-weighted image showed the cyst wall to be attached to the knee joint capsule. tial diagnosis of cysts at proximal calf. Based on the site of origin, intramuscular cysts can be divided into the following five types: intramuscular cysts arising from the muscular tissue, knee joint, PTFJ, neurilemma and PVNS. The characteristics of these cysts but cysts arising from PVNS resemble the characteristics of a ganglion; hence, these cysts can be considered as intramuscular ganglion cysts.

The ganglion cyst is a benign cystic mass, with a dense fibrous connective tissue capsule, containing clear, high-viscosity mucinous fluid [15], and usually originate from the joint capsule or tendon sheath. The pathogenesis of ganglia is controversial. Rohrich et al [16] mentioned that there were three predominant theories about the origin of a ganglion: 1) they arise as an outpouching or weakening of the joint capsule, 2) they arise from a mesothelial embryonic rest, and 3) they arise due to mucin formation by fibroblasts of the joint space or bursa. In an extensive review of the literature, we found that ganglion cysts occur most frequently in juxta-articular locations such as hand and wrist $[17,18]$. However, intramuscular ganglion cysts are uncommon, with only a few reported cases [3-14, 16, 19-25], of which, intramuscular ganglion cysts located in the proximal calf are even rarer with fewer case reports $[3-14,19,24,25]$.

For accurate diagnosis and appropriate treatment, we present below, a precise description of these cysts at the proximal calf.

\section{Intramuscular cysts arising from the muscular tissue}

We defined the intramuscular ganglion cyst, which was not attached to the adjacent joint, as an intramuscular cyst arising from the muscular tissue. This type of cyst is currently rare. Rohrich et al [16] first reported a patient with a ganglion within the biceps muscle, which had no association with adjacent joints. They postulated that the ganglion might have originated from embryonic rest of ectopic synovial tissue. Subsequently, James et al [26] presented a series of 10 cases of ganglion cysts arising from the medial or lateral gastrocnemius muscle. No link could be identified between the cyst and knee joint or any other intra-articular structure on MRI. However, none of them was dissected intramuscularly. Beggs et al [19] presented the imaging findings in three patients who had intramuscular 
ganglia, which were not attached to a joint, and one case had the ganglion in the medial gastrocnemius muscle. Park et al [25] reported a case of ruptured intramuscular ganglion cyst in the gastrocnemius medialis muscle. Nicholson et al [24] also described a case of intramuscular ganglion cyst in the medial gastrocnemius muscle. The cysts in these two cases were not attached to the knee joint on MRI, and histopathology confirmed the diagnosis of ganglion cysts. The first case in our article is also an intramuscular ganglion cyst at the proximal calf. MRI showed no communication between the cyst and the adjacent joint. Arthroscopic examination was performed and we did not find any evidence of intra-articular pathology or capsular opening. The histological diagnosis was ganglion cyst. Based on previous case reports, we deduced that these intramuscular ganglion cysts arise from the muscular tissue. For this type of cysts, satisfactory clinical results can be achieved by direct open excision $[19,24,25]$.

\section{Intramuscular cysts arising from the knee joint}

When the intramuscular cysts communicate with the joint cavity through a tortuous pedicle, they are considered to be arising from the joint. These types of cysts arise as an outpouching or weakening of the joint capsule [16]. In addition, Fang et al [3] thought that current knowledge about the anatomy and pathophysiology of popliteal cysts does not convincingly explain the mechanism by which intramuscular cysts arise. They presumed that cysts extend along the intermuscular planes that offer least resistance. Chiu et al [20] reported the case of a child with an intramuscular cyst in the thenar eminence, with its origin in the scaphocapitate joint. Montet et al [23] also reported a case of intramuscular ganglion arising from the acromioclavicular joint. Intramuscular cysts arising from the knee joint capsule can be considered as atypical Baker's cysts. Baker's cyst is a fluid-filled mass, which is a distention of the gastrocnemio-semimembranosus bursa, located on the medial aspect of popliteal fossa. Several previous studies have shown the communication between the gastrocnemio-semimembranosus bursa and the joint cavity [27-29]. This plays an important role as a diagnostic marker for the differentiation between extra-articular soft tissue ganglion cyst and Baker's cyst.

Intramuscular Baker's cysts have rarely been reported in the international literature. Kim et al [30] reported a case of Baker's cyst with intramuscular extension into the vastus medialis muscle. Kim et al [4] reported a ganglion cyst in gastrocnemius muscle, which had a channel communicating with the knee joint. Fang et al [3] described the intramuscular dissection of Baker's cysts in three cases. An extension of a Baker's cyst was shown to involve the vastus medialis in one case and two cases involved the medial head of gastrocnemius. In each of these reports, a channel was seen to be communicating with the knee joint on MRI, allowing identification of the source of these cysts. Clearly, it is important to differentiate atypical Baker's cysts from ganglion cysts arising close to the medial head of gastrocnemius that this study describes. Historically, direct open excision has resulted in unacceptably high recurrence rates, as the intra-articular pathology that causes and sustains the cyst is not curable and the communication with the joint is not tightly closed [31-33]. Currently, arthroscopic procedures are most commonly used to treat the intra-articular pathology and to address the cysts directly by opening [32] or closing [34] the orifice of communication. However, we propose that open surgery combined with arthroscopic procedures is also necessary when the cysts are large.

\section{Intramuscular cysts arising from the PTFJ}

Similarly, when intramuscular cysts communicate with the PTFJ cavity, they are considered as arising from the PTFJ. In one case reported by Brooks [35], the ganglion invaded the tibialis anterior muscle. Subsequently, Muckart [7] described eight patients with ganglia arising from the superior tibiofibular joint, five of which were within the peroneus longus muscle and were connected to the superior tibiofibular joint, through the tendinous origin of the muscle. The clinical presentation of this type of cysts is variable, from asymptomatic to gradually increasing swelling and pain. In addition, it is known that over $50 \%$ of cysts of the PTFJ are associated with peroneal nerve palsy, due to compression of the common peroneal nerve in the peroneal canal [5-8, 35-37]. This is characterized by anterolateral leg and dorsal foot pain, followed by progressive weakness of the peroneal nerve innervated anterior compartment musculature, along with a steppage gait. Complete peroneal nerve palsy presenting with a foot drop has been reported [13, 14, 38]. We believe that MRI is the best imaging method for the diagnosis and differential diagnosis of a cyst arising from the PTFJ. It provides the best images of the communicating channel leading from the cyst into the PTFJ, and proximity of the cyst to the peroneal nerve $[8,15]$. Aspiration and corticosteroid injection in the cyst has limited success and surgical excision with removal of the pedicle connecting the cyst to the joint is recommended to reduce the risk of recurrence $[8,14,39]$.

\section{Intramuscular cysts arising from the neurilemma}

When ganglions in intra-neural locations occur within the sheaths of peripheral nerves, they are called nerve sheath ganglions $[10,12]$. Nerve sheath ganglions are most commonly found in peroneal or tibial nerve in the lower limb $[12,40]$ or ulnar nerve in the upper extremity $[9,11]$. This type of intramuscular ganglion is called an intramuscular cyst arising from the neurilemma. The etiology of these nerve sheath ganglions is controversial. Patel et al [41] reported a case of fascicular posterior tibial nerve involvement by a ganglion cyst in the calf, which they presumed was a perineural pseudoganglion cyst, and agreed with the hypothesis that repetitive nerve compression was the etiology. However, some cases in the literature do not involve clear differentiation between intramuscular cysts arising from the PTFJ and intramuscular cysts arising from the neurilemma, and they were believed to be the same entity [13-15]. These cysts usually present as tender masses with pain or numbness in the distribution of the affected nerve, due to nerve compression and nerve involvement. Similarly, MRI can be used to document the cystic nature and extent of the mass. In addition, electromyographic examination can also 
reveal the involved nerve as well as the extent of nerve damage $[40,42]$. A complete surgical removal of the cyst is the most reliable treatment. However, safe removal of the ganglion cannot be achieved without compromising the nerve's integrity. Therefore, incising the cyst wall, drainage of the gelatinous contents and injection of steroids to prevent recurrence, while leaving the cyst wall left open, appeared to be effective in treating these cases [12].

\section{Intramuscular cysts arising from the PVNS}

PVNS, which was first described by Jaffe et al in 1941 [43], is rare, with an annual incidence of 1.8 cases per million [44]. It is a benign but aggressive pathology of the synovium, often localized to the knee joint $[45,46]$. It is characterized by synovial proliferation and hemosiderin deposition inside the joints, tendon sheaths, and bursae. Granowitz et al [47] further sub classified them into diffuse and localized types, and the diffuse type is reportedly more common than the localized type [44]. Similarly, its etiology is also controversial. The clinical presentation includes periarticular pain and swelling [45]. MRI is the best imaging method for diagnosis, but definitive diagnosis can only be confirmed by pathological biopsy. The management of PVNS includes open surgical excision and arthroscopic synovectomy, which have been reported to have a high rate of recurrence $[48,49]$.

Extra-articular spread of PVNS in the knee joint has scarcely been reported. Tatari et al [50], Gokhale et al [51] and Tosti et al [52] each described a case of PVNS in the popliteal fossa presenting as a popliteal cyst, and concluded that the disease had originated in the knee and spread to the popliteal space. However, after a thorough systematic literature review, we found that this is the first case report about intramuscular cysts at proximal calf arising from the PVNS. In this case, the patient's primary clinical manifestation is pain in the knee joint and swelling due to PNVS of the joint, without any symptoms of an intramuscular cyst. MRI provides a clear image of the cyst's location and communication between the cyst and knee joint. Compared to intramuscular cysts arising from a different etiology, this type of cyst has a thickened wall with low signal intensity on both T1-weighted and T2-weighted images. Due to the absence of clinical manifestations, we did not perform any clinical intervention for the cyst. However, in case of compression of the adjacent nerve and vessel or limitation of the range of knee joint movements, we suggest that both arthroscopic synovectomy and cyst excision are necessary.

In conclusion, depending on the origin of intramuscular cysts, they were divided into the following five types: intramuscular cysts arising from the muscular tissue, knee joint, PTFJ, neurilemma and PVNS. The therapy varies based on the origin; hence, it is important to identify the origin of the cyst.

\section{Acknowledgments}

The authors are grateful to the staff of the Department of Or- thopedics, Beijing Shunyi District Hospital for their valuable assistance with in collection of the patients' medical records.

\section{Conflict of Interest}

None of the authors has any potential conflict of interest.

\section{References}

1. Krudwig WK, Schulte KK, Heinemann C. Intra-articular ganglion cysts of the knee joint: a report of 85 cases and review of the literature. Knee Surg Sports Traumatol Arthrosc. 2004;12(2):123-129.

2. Marti-Bonmati L, Molla E, Dosda R, Casillas C, Ferrer P. MR imaging of Baker cysts - prevalence and relation to internal derangements of the knee. MAGMA. 2000;10(3):205-210.

3. Fang CS, McCarthy CL, McNally EG. Intramuscular dissection of Baker's cysts: report on three cases. Skeletal Radiol. 2004;33(6):367-371.

4. Kim JY, Jung SA, Sung MS, Park YH, Kang YK. Extraarticular soft tissue ganglion cyst around the knee: focus on the associated findings. Eur Radiol. 2004;14(1):106111.

5. Evans JD, Neumann L, Frostick SP. Compression neuropathy of the common peroneal nerve caused by a ganglion. Microsurgery. 1994;15(3):193-195.

6. Gayet LE, Morand F, Goujon JM, Pries P, Clarac JP. Compression of the peroneal nerve by a cyst in a sevenyear-old child. Eur J Pediatr Surg. 1998;8(1):61-63.

7. Muckart RD. Compression of the common peroneal nerve by intramuscular ganglion from the superior tibio-fibular joint. J Bone Joint Surg Br. 1976;58(2):241-244.

8. Pecina HI, Boric I, Pecina TC, Smoljanovic T, Pecina M. Double synovial cyst of the proximal tibiofibular joint confirmed by MRI as a cause of the peroneal tunnel syndrome. Acta Chir Orthop Traumatol Cech. 2008;75(4):301-305.

9. Chick G, Alnot JY, Silbermann-Hoffman O. Intraneural mucoid pseudocysts. A report of ten cases. J Bone Joint Surg Br. 2001;83(7):1020-1022.

10. Harbaugh KS, Tiel RL, Kline DG. Ganglion cyst involvement of peripheral nerves. J Neurosurg. 1997;87(3):403408.

11. Reynolds DL, Jr., Jacobson JA, Inampudi P, Jamadar DA, Ebrahim FS, Hayes CW. Sonographic characteristics of peripheral nerve sheath tumors. AJR Am J Roentgenol. 2004;182(3):741-744.

12. Tseng KF, Hsu HC, Wang FC, Fong YC. Nerve sheath ganglion of the tibial nerve presenting as a Baker's cyst: a case report. Knee Surg Sports Traumatol Arthrosc. 2006;14(9):880-884

13. Damron TA, Rock MG. Unusual manifestations of proximal tibiofibular joint synovial cysts. Orthopedics. 1997;20(3):225-230.

14. Jerome D, McKendry R. Synovial cyst of the proximal tibiofibular joint. J Rheumatol. 2000;27(4):1096-1098. 
15. McCarthy CL, McNally EG. The MRI appearance of cystic lesions around the knee. Skeletal Radiol. 2004;33(4):187-209.

16. Rohrich RJ, Rich BK. Intramuscular ganglion of the biceps muscle. Ann Plast Surg. 1994;33(4):432-433.

17. Bianchi S, Abdelwahab IF, Zwass A, Giacomello P. Ultrasonographic evaluation of wrist ganglia. Skeletal Radiol. 1994;23(3):201-203.

18. Thornburg LE. Ganglions of the hand and wrist. J Am Acad Orthop Surg. 1999;7(4):231-238.

19. Beggs I, Saifuddin A, Limb D. Non-communicating intramuscular ganglia. Eur Radiol. 1998;8(9):1657-1661.

20. Chiu DT, Ascherman JA. An intramuscular ganglion presenting as a thenar mass. Plast Reconstr Surg. 1994;93(3):606-607.

21. Jackson L, Namey TC. Ganglion cyst within the quadriceps muscle: evaluation with computed tomography and ultrasound. A case report. Orthopedics. 1987;10(8):11791180.

22. Kim YJ, Chae SU, Choi BS, Kim JY, Jo HJ. Intramuscular ganglion of the quadriceps femoris. Knee Surg Relat Res. 2013;25(1):40-42.

23. Montet X, Zamorani-Bianchi MP, Mehdizade A, Martinoli C, Bianchi S. Intramuscular ganglion arising from the acromioclavicular joint. Clin Imaging. 2004;28(2):109112.

24. Nicholson LT, Freedman HL. Intramuscular dissection of a large ganglion cyst into the gastrocnemius muscle. Orthopedics. 2012;35(7):e1122-1124.

25. Park S, Jin W, Chun YS, Park SY, Kim HC, Kim GY, Park JS, et al. Ruptured intramuscular ganglion cyst in the gastrocnemius medialis muscle: sonographic appearance. J Clin Ultrasound. 2009;37(8):478-481.

26. James SL, Connell DA, Bell J, Saifuddin A. Ganglion cysts at the gastrocnemius origin: a series of ten cases. Skeletal Radiol. 2007;36(2):139-143.

27. Rauschning W. Anatomy and function of the communication between knee joint and popliteal bursae. Ann Rheum Dis. 1980;39(4):354-358.

28. Lindgren PG, Willen R. Gastrocnemio-semimembranosus bursa and its relation to the knee joint. I. Anatomy and histology. Acta Radiol Diagn (Stockh). 1977;18(5):497512.

29. Lindgren G, Rauschning W. Clinical and arthrographic studies on the valve mechanism in communicating popliteal cysts. Arch Orthop Trauma Surg. 1979;95(4):245250 .

30. Kim TW, Suh JT, Son SM, Moon TY, Lee IS, Choi KU, Kim JI. Baker's cyst with intramuscular extension into vastus medialis muscle. Knee Surg Relat Res. 2012;24(4):249-253.

31. Rauschning W, Lindgren PG. Popliteal cysts (Baker's cysts) in adults. I. Clinical and roentgenological results of operative excision. Acta Orthop Scand. 1979;50(5):583591.

32. Sansone V, De Ponti A. Arthroscopic treatment of popliteal cyst and associated intra-articular knee disorders in adults. Arthroscopy. 1999;15(4):368-372.

33. Takahashi M, Nagano A. Arthroscopic treatment of pop- liteal cyst and visualization of its cavity through the posterior portal of the knee. Arthroscopy. 2005;21(5):638.

34. Calvisi V, Lupparelli S, Giuliani P. Arthroscopic allinside suture of symptomatic Baker's cysts: a technical option for surgical treatment in adults. Knee Surg Sports Traumatol Arthrosc. 2007;15(12):1452-1460.

35. Brooks DM. Nerve compression by simple ganglia. J Bone Joint Surg Br. 1952;34-B(3):391-400.

36. Gibbon AJ, Wardell SR, Scott RD. Synovial cyst of the proximal tibiofibular joint with peroneal nerve compression after total knee arthroplasty. J Arthroplasty. 1999;14(6):766-768.

37. Hersekli MA, Akpinar S, Demirors H, Ozkoc G, Ozalay M, Cesur N, Uysal M, et al. Synovial cysts of proximal tibiofibular joint causing peroneal nerve palsy: report of three cases and review of the literature. Arch Orthop Trauma Surg. 2004;124(10):711-714.

38. Stener B. Unusual ganglion cysts in the neighbourhood of the knee joint. A report of six cases - three with involvement of the peroneal nerve. Acta Orthop Scand. 1969;40(3):392-401.

39. Mortazavi SM, Farzan M, Asadollahi S. Proximal tibiofibular joint synovial cyst - one pathology with three different presentations. Knee Surg Sports Traumatol Arthrosc. 2006;14(9):875-879.

40. Coleman SH, Beredjeklian PK, Weiland AJ. Intraneural ganglion cyst of the peroneal nerve accompanied by complete foot drop. A case report. Am J Sports Med. 2001;29(2):238-241.

41. Patel C, Vishnubhakat SM, Narayan R. Fascicular involvement of the posterior tibial nerve as a result of perineural ganglion cyst at the posterior tibial nerve in the calf: a case report and review of literature. J Clin Neuromuscul Dis. 2015;17(2):84-87.

42. Antonini G, Bastianello S, Nucci F, Artico M, Bozzao L, Millefiorini M. Ganglion of deep peroneal nerve: electrophysiology and CT scan in the diagnosis. Electromyogr Clin Neurophysiol. 1991;31(1):9-13.

43. Jaffe HL, Lichtenstein L, Sutro CJ. Pigmented villonodular synovitis, bursitis and tenosynovitis. Arch Pathol. 1941;31:731-765.

44. Myers BW, Masi AT. Pigmented villonodular synovitis and tenosynovitis: a clinical epidemiologic study of 166 cases and literature review. Medicine (Baltimore). 1980;59(3):223-238

45. Ottaviani S, Ayral X, Dougados M, Gossec L. Pigmented villonodular synovitis: a retrospective single-center study of 122 cases and review of the literature. Semin Arthritis Rheum. 2011;40(6):539-546.

46. Xie GP, Jiang N, Liang CX, Zeng JC, Chen ZY, Xu Q, Qi RZ, et al. Pigmented villonodular synovitis: a retrospective multicenter study of 237 cases. PLoS One. 2015;10(3):e0121451.

47. Granowitz SP, D'Antonio J, Mankin HL. The pathogenesis and long-term end results of pigmented villonodular synovitis. Clin Orthop Relat Res. 1976114):335-351.

48. Gu HF, Zhang SJ, Zhao C, Chen Y, Bi Q. A comparison of open and arthroscopic surgery for treatment of diffuse pigmented villonodular synovitis of the knee. Knee Surg 
Sports Traumatol Arthrosc. 2014;22(11):2830-2836.

49. Jain JK, Vidyasagar JV, Sagar R, Patel H, Chetan ML, Bajaj A. Arthroscopic synovectomy in pigmented villonodular synovitis of the knee: clinical series and outcome. Int Orthop. 2013;37(12):2363-2369.

50. Tatari H, Baran O, Lebe B, Kilic S, Manisali M, Havitcioglu H. Pigmented villonodular synovitis of the knee pre- senting as a popliteal cyst. Arthroscopy. 2000;16(6):13.

51. Gokhale N, Purohit S, Bhosale PB. Pigmented villonodular synovitis presenting as a popliteal cyst. J Orthop Case Rep. 2015;5(3):63-65.

52. Tosti R, Kelly JDt. Pigmented villonodular synovitis presenting as a baker cyst. Am J Orthop (Belle Mead NJ). 2011;40(10):528-531. 